\title{
Perception of depth: Processing of simple positional disparity as a function of viewing distance
}

\author{
MANFRED RITTER \\ Philipps-Universität Marburg, Gutenbergstrasse 18, D-3550 Marburg, West Germany
}

\begin{abstract}
Dependency of perceived depth (relative to the fixation point) on disparity, viewing distance, and the type of the stereoscopic stimulus was investigated. Nearly complete constancy of depth, as required for a veridically matched perception, was observed only at small disparity values and with the larger square-formed stimulus; under these conditions, perceived depth corresponded well with real depth intervals for close viewing distances. Additionally, a model for perceptual processing of both variables, disparity and viewing distance, was applied to the data.
\end{abstract}

When observing one's surroundings, one usually looks from one object to another, and in this sense the desired stimulus array is sampled by means of voluntary eye movements (cf. Foley \& Richards, 1972). Then, according to rule, space perception is derived from several such stimulus samples. The spatial information thereby extracted by the visual system includes egocentric or absolute direction and distance (i.e., object direction and object distance relative to the observer), relative directions and distances (i.e., differences in directions and distances between several objects) and spatial depth of the objects themselves. If space is to be perceived veridically, then in stimulus processing the latter two aspects have to be scaled using the absolute values as a base. In the following experiments, a subproblem of this complex was investigated, namely the perception of a spatial depth interval based on the interaction of simple positional disparity and viewing distance.

Compared to relative positional disparity and orientational disparity (Blakemore, 1970; Blakemore, Fiorentini, \& Maffei, 1972; von der Heydt \& Adorjani, Note 1), the so-called simple positional disparity is considered the most basic form of disparity; it signals a single depth interval, $d$, between one object, $O$, and the point of fixation, $F$, which lies in a viewing distance, D, from the observer's eyes. Figure 1 schematically shows the relation between simple positional disparity (henceforth called "disparity"), depth interval, $\mathrm{d}$, viewing distance, $\mathrm{D}$, and interocular distance, $i$. Because the object point, $O$, and the fixation point, $F$, both lie on a line perpendicular to the midpoint of the interocular distance, disparity can be described by one single angular magnitude, $\gamma$; supposing a symmetrical eye position and that $\mathrm{D}$ is much larger than $i$ and $d$, then the following relation between these variables exists:

$$
r=\left(\frac{\mathrm{i}}{2}\right) \frac{\mathrm{d}}{\mathrm{D}^{2}} ; \quad(\mathrm{rad})
$$

Thus, when an observer looks at the same spatial depth interval from different viewing distances, the magnitude of retinal disparity is inversely proportional to the square of the viewing distance; because the interocular distance is constant, it can be neglected from now on. Complete depth constancy implies that a physical depth interval, at which an observer views from different distances, is perceived veridically and, in this sense, is independent of viewing distance. This requires that a perceptual process neutralizes the stimulus transformation which is imposed on the physical depth interval (distal stimulus) while being looked at. By means of such a correction process, perception approximates the value of the distal stimulus.

In the following experiments, depth constancy was investigated under the simplest possible stimulus conditions. (1) Ritter (1977) and Wallach and

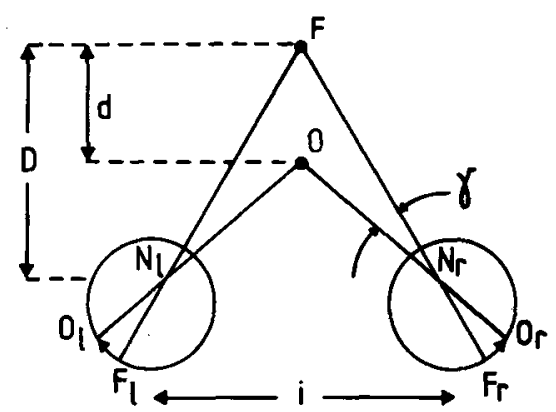

Figure 1. Simple positional disparity, $\gamma$, as a function of viewing distance, $\mathbf{D}$, for a depth interval, $d$, and an interocular distance, $i$, at a symmetrical eye position. 
Zuckerman (1963) had shown that depth constancy is almost complete for close distances (up to about $2 \mathrm{~m}$ ) (see also Ono \& Cumerford, 1977). In these reports, geometrical objects or their shadow outlines were used as stimuli, therefore causing intermixed disparity conditions; secondly, the comparison tasks had no time limit, so eye movements could influence the perceptual performance (Foley \& Richards, 1972). Therefore, in the following experiments, the stereoscopic stimuli were simplified and presented for such a short duration that no active eye movement could be performed during presentation. (2) The determination of viewing distance by the perceptual system should be restricted, if possible, to one stimulus indicator only. Eye convergence appears to be most important at near distances (Gogel, 1977; Owens \& Leibowitz, 1976; von Hofsten, 1976). In an experimental situation similar to that used below, it was shown, by dissociating the two distance cues, accommodation and convergence, that the visual system relies on the convergence value only in this case of distance determination (Ritter, 1977). (3) Foley and Richards (1972) found that (for a specific viewing distance) perceived depth is an inverse U-shaped function of disparity, having its maximum value at about $40-60 \mathrm{~min}$ of arc and decreasing to zero at about $120 \mathrm{~min}$. However, these findings disagree with results of Blakemore (1970) and Westheimer and Tanzman (1956), who found depth perception at much larger disparity values. (4) Since Richards and Kaye (1974) could demonstrate that the size of the stereoscopic stimuli can influence the range of depth perception, this variable was also included.

Thus, the following three experiments were designed: First, how complete the constancy of stereoscopic depth is under the simplest stimulus conditions was investigated; for this purpose, disparity was kept constant at a small value and the relation between perceived depth and viewing distance was determined (Experiment 1). Second, the interaction between disparity and viewing distance was analyzed to learn if it could be described by means of one single connecting function. In this case, it must be shown that depth values for different disparities and stimulus configurations conform to this assumption; accordingly, perceived depth was investigated as a function of disparity and stimulus configuration at two viewing distances (Experiment 2). Both above experiments presume that perceived depth or perception of relative distance can be validly measured; Experiment 3 served this purpose.

\section{EXPERIMENT 1}

In Experiment 1, the magnitude of depth constancy was determined. According to the above stimulus analysis, it could be expected that, for complete con- stancy, perceived depth (relative to the fixation point) was a quadratic function of viewing distance. To what extent this is valid was examined. Thus, this experiment further checked the results of a previous one (Ritter, 1977), though now under more simple conditions of depth perception.

\section{Method}

Subjects. The subjects were students who were first tested with the Roda-Test (manufactured by G. Rodenstock, München). All six selected subjects had a near and far visual acuity equivalent better than $1 \mathrm{~min}$ of arc and a stereoscopic acuity equivalent better then $22.5 \mathrm{~min}$ of arc.

Apparatus and Stimuli. The subjects looked at a translucent screen through Polaroid filters, a beam-splitting mirror, and a rectangular opening in a screen set $14 \mathrm{~cm}$ in front of the subject's eyes. The area of the visible projection screen was $40 \times 12 \mathrm{deg}$. By means of two projectors, a homogeneous background luminance of $30 \mathrm{~cd} / \mathrm{m}^{2}$ and a fixation target having a luminance of $150 \mathrm{~cd} / \mathrm{m}^{2}$ were produced; a further double projector with appropriately mounted Polaroid filters and electromagnetic shutters in front of the lenses presented the stereoscopic stimuli $\left(150 \mathrm{~cd} / \mathrm{m}^{2}\right)$. The subject could see the stimuli straight ahead and at eye level. On a track lying at an oblique direction to the subject's line of sight, a small circular target serving as a probe (comparison stimulus) could be moved by the experimenter; its position on the track could be read off in millimeters. By means of the beam splitter, the comparison stimulus was superimposed onto the main direction of stimulus presentation (see Figure 2). Time sequence and duration of the stimulus presentation were controlled by digital modular components (Marburger System; Kalveram, Note 2). Using a microswitch, the subject could start the presentations. A chin- and foreheadrest was used to minimize head movements.

Three different stimuli were used: a fixation target, the stereoscopic stimuli, and the comparison stimulus (see Figure 3 ). The fixation target had a rectangular form $(.75 \times 3.5 \mathrm{deg})$; at the middle of its lower edge, a triangular tip to be fixated at its apex was inserted. The comparison stimulus had a circular form with a diameter of $.5 \mathrm{deg}$. The stereoscopic stimuli were square-

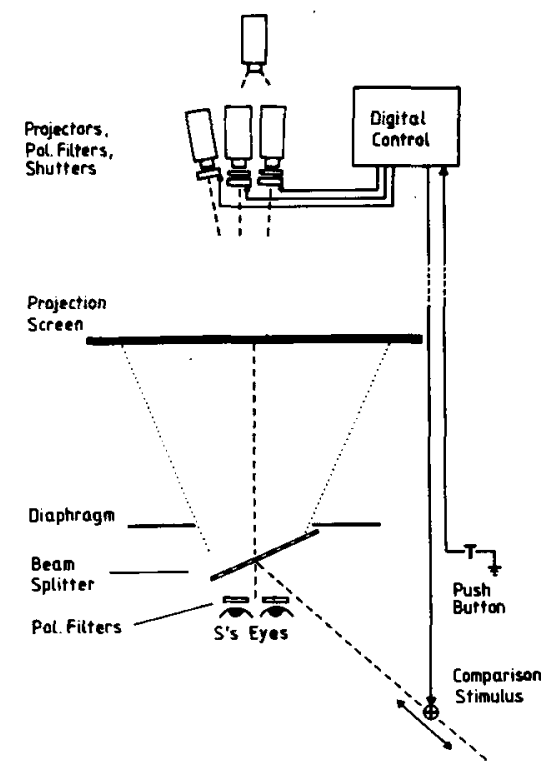

Figure 2. Schematic diagram of the experimental arrangement. 


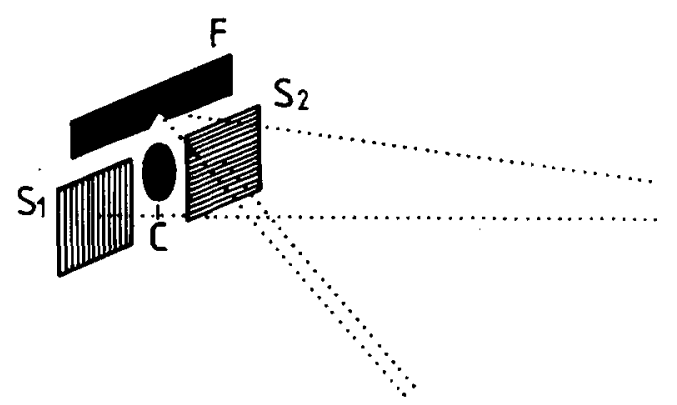

Figure 3. Arrangement of the stimuli presented: fixation target, $F$, stereoscopic stimuli, $S_{1}$ and $S_{2}$, and comparison stimuli, $C$.

formed $(1 \times 1 \mathrm{deg})$. Disparity was held constant, its value being $20 \mathrm{~min}$ of arc (as related to the midpoints of the two squares); its direction was convergent, i.e., the stimuli were normally seen by the subject in the range between his eyes and the fixation target. The distance between the two stereoscopic stimuli on the projection screen, necessary to generate specific disparity magnitudes, were computed to their exact values without the simplifying assumptions employed above. Thus, a mean distance of $65 \mathrm{~mm}$ between the center of rotation of both eyes and a distance of $6 \mathrm{~mm}$ between the nodal point and the center of rotation of one eye were assumed (Gulick \& Lawson, 1976). The same values were substituted in computing the magnitude of complete depth constancy.

During one single trial, the fixation target was first presented for $2.2 \mathrm{sec}$; the stereoscopic stimuli were presented for $100 \mathrm{msec}$, ending exactly at the same time as the fixation stimulus and thereby excluding active eye movements during stimulus input. One second later, the comparison stimulus was shown for $2 \mathrm{sec}$. Between trials, there was a pause of about 6 to $10 \mathrm{sec}$.

Viewing distance served as the independent variable; it was varied in gradations of $60,90,120,150$, and $180 \mathrm{~cm}$. In control trials, only the fixation target and the comparison stimulus were presented; in experimental trials, the stereoscopic stimuli were also included.

Procedure. The subject's task was to look at the fixation target and, according to instructions, to remember the egocentric distance of the fixation target or that of the stereoscopic stimuli. Then the subject looked at the comparison stimulus and could instruct the experimenter in which direction the probe should be moved to correspond with the position of the stimulus. Trials were repeated until a complete match was reached. One such measurement comprised four to eight successive trials. A weighted difference between the subjects' settings of the probe for the fixation target and for the stereoscopic stimuli served as the value of perceived depth. (See Experiment 3.)

For each value of viewing distance, four measurements were taken for the perceived distance of the fixation target and four for the stereoscopic stimulus. Each subject was tested in all experimental conditions. The order of sequence of the viewing distances was varied across subjects.

\section{Results}

Figure 4 shows the relation between perceived depth and viewing distance when disparity was held constant. An increase of viewing distance results in an increase of perceived depth. The dashed line in the figure corresponds to the values expected for complete depth constancy. Observed and theoretically expected values agreed well, and only for the largest value of viewing distance was perceived depth smaller than expected $[\mathrm{t}(6)=5.07 ; \mathrm{p}<.01]$. Hence, nearly complete depth constancy can also be reached under simple stimulus conditions. Thus, it can be concluded that the visual system obtains perceived depth (relative to the fixation point) from the processing of both disparity and viewing distance.

\section{EXPERIMENT 2}

The simplest model for depth perception which describes the processing of both variables, disparity and viewing distance, assumes a processing instance in which the internal afferent message $\gamma^{\prime}$ is multiplied by a magnitude corresponding to the square of the internal distance message, $D^{\prime}$ (see Figure 5). Before acting as proximal stimulus, a distal physical depth interval, $\mathrm{d}$, is divided by the square of the actual viewing distance, $\mathrm{D}$, according to geometrical laws. Because $\gamma^{\prime}$ systematically depends on $\gamma$ and $D$, the visual system can obtain d' by a kind of a "reverse" process, namely, by multiplying $\gamma^{\prime}$ with $\mathrm{D}^{\prime}$, the internal distance message. This can be considered as one example of a perceptual correcting process which may be called cancellation (cf. Bischof, 1966, 1974): An internal process neutralizes the effect which is imposed on the distal stimulus during its obervation, and thus perception remains veridical. In the simplest case, for this example, the same neutralizing function applies for various disparity conditions.

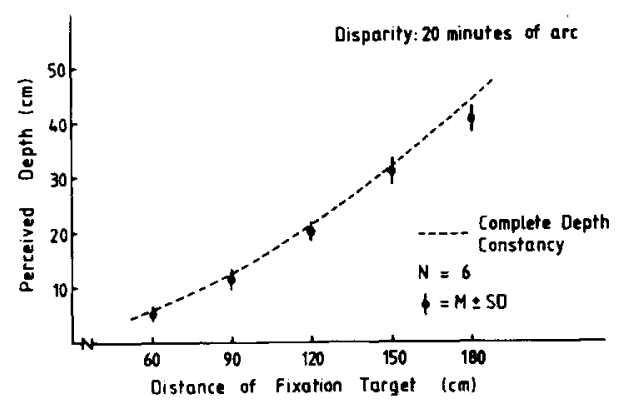

Figure 4. Perceived depth (relative to the point of fixation) at constant disparity as a function of viewing distance; means and standard deviations of six subjects. The dashed line signifies the values of complete depth constancy.

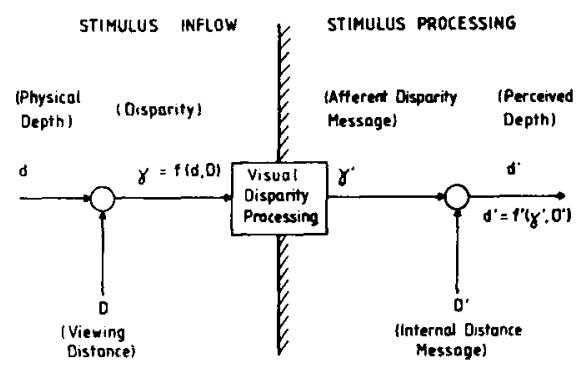

Figure 5. Schematic diagram of the model assumed for processing disparity and viewing distance. The hatched line divides stimulus inflow from stimulus processing. 
The data of Experiment 1 are compatible with such an assumption. In order to test its validity in a larger range of conditions, the following experiment served to measure depth constancy as a function of disparity, of the type of stereoscopic stimuli, and of viewing distance. The obtained data were also analyzed according to what extent they could be described by the above model.

\section{Method}

The same experimental apparatus and procedure as in Experiment 1 were used. Disparity, viewing distance, and stimulus type served as independent variables. Disparity was varied in the stages of $10,20,40,60,80,100,120,140$, and $160 \mathrm{~min}$ of arc; its direction was convergent. Two different forms of stereoscopic stimuli were used, a vertical line stimulus $(2 \mathrm{~min} \times 1 \mathrm{deg})$ and a square stimulus $(1 \times 1 \mathrm{deg})$. Viewing distance was varied in two values, 90 and $150 \mathrm{~cm}$.

The subject's task was the same as in Experiment 1; in addition, the subject had to judge whether he could see either one single fused stimulus or definitely two stimuli, or if no decision could be made between these two possibilities. For this qualitative judgment, only those trials were used in which a match between test and comparison stimulus was reached. This should serve as an additional description of depth perception in different conditions.

Seven subjects participated in the experiment; their visual and stereoscopic vision were normal (Roda-Test). Each subject was tested in all conditions; their order of sequence was varied across subjects.

\section{Results}

Figure 6 shows the means and standard deviations of perceived depth as a function of disparity and stimulus type for two viewing distances; the dashed

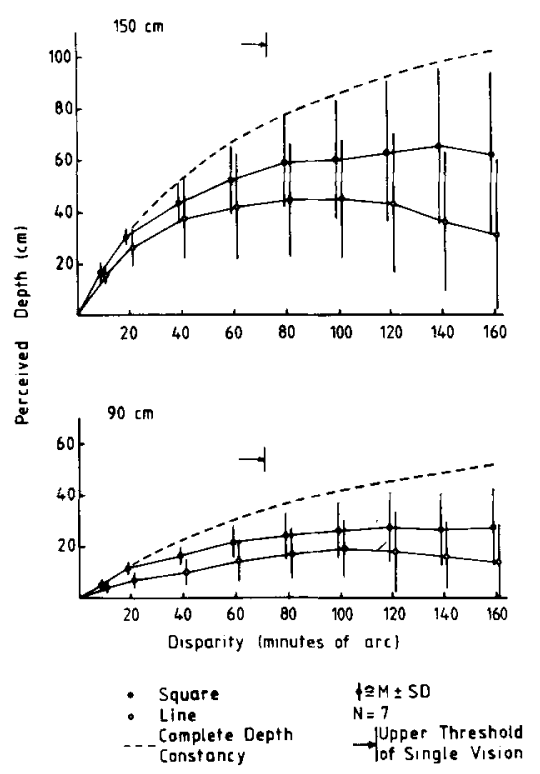

Figure 6. Perceived depth (relative to the point of fixation) as a function of disparity and type of stimulus for two viewing distances; means and standard deviations for seven subjects. The arrow indicates up to which mean disparity value the stimulus was seen as a single one. line corresponds to values of perceived depth expected for complete depth constancy. The magnitude of perceived depth proved to be an inverse U-shaped function of disparity, its range being larger for the square stimulus. A good correspondence with complete depth constancy values could be obtained only for small disparities and the square stimulus. The increase of the standard deviations with increasing disparities is caused by large interindividual differences in the range of disparities for which different subjects could see stimuli in depth (cf. Richards, 1970).

An analysis of variance showed that besides the main effects and the first-order and second-order interaction terms, the $F$ value of the third-order interaction between Disparity, Viewing Distance, and Stimulus Type was also significant $[\mathrm{F}(8,48)=4.1$; $\mathrm{p}<.01]$. Perceived depth is therefore differentially dependent on disparity, viewing distance, and type of stimulus. Both stimuli, line and square-this was the result of the qualitative judgment-were seen as a single fused stimulus up to a mean disparity value of $70 \mathrm{~min}$ of arc.

In the above model, it is assumed that perceived depth, $d^{\prime}$, is based on one single processing function relating $\gamma^{\prime}$ and $D^{\prime}$. If this is valid, it should be demonstrable that the magnitude of the afferent disparity message, $\gamma^{\prime}$, computed back from the experimental data, is dependent only on disparity $\gamma$ and the type of stimulus, and not on viewing distance. To prove this, the experimental data were transformed according to the function

$$
\gamma^{\prime}=\left(\frac{\mathrm{i}}{2}\right) \frac{\mathrm{d}^{\prime}}{\mathrm{D}^{\prime 2}}
$$

(The description is simplified here; the exact trigonometric relations were used for computation.) As a first approximation, $\mathrm{D}^{\prime}$ is substituted with $.87 \mathrm{D}$, a value taken from Experiment 3. Figure 7 shows the mean values of this hypothetical magnitude; it clearly demonstrates the expected independence of viewing distance. (In an analysis of variance, therefore, none of the $F$ values, neither in the Viewing Distance main effect nor in its interactions with the other factors proved to be significant.)

This result is compatible with the hypothesis that to attain perception of depth the afferent disparity message $\gamma^{\prime}$ is transformed according to one single function. The inverse U-shaped functions obtained for $\gamma^{\prime}$ were dependent on the stimulus type. These functions were much larger as compared to tuning curves for single disparity-detecting neurons (Bishop, 1973; Pettigrew, Nikara, \& Bishop, 1968). It is therefore conceivable that $y^{\prime}$ is the result of integrating the output of several such neuronal networks, each differentially sensitive for a small disparity range. 


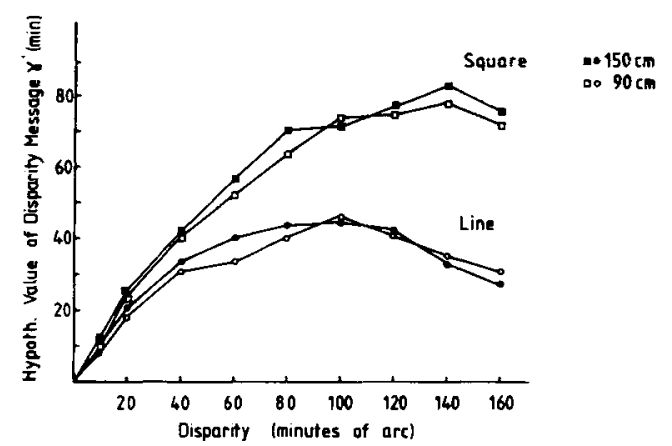

Figure 7. Hypothetical values of the afferent disparity message, computed back from the experimental data of Figure 6 , as a function of disparity, stimulus type, and viewing distance.

\section{DISCUSSION}

This report furnishes the following results: (1) Constancy of stereoscopic depth was also obtained for small disparities after restricting the available stimulus information to that of one single fixation; perceived depth (relative to the point of fixation) corresponded well with real depth intervals, which cause such disparities in a natural viewing condition when changing viewing distance; this is maintained up to a viewing distance of about $150 \mathrm{~cm}$ (Experiment 1). (2) The variation of viewing distance, disparity, and type of stimulus resulted in variation of perceived depth. The relationship between perceived depth and disparity was found to be an inverse U-shaped function for both stimulus forms, line and square, and both viewing distances. Complete depth constancy was obtained with only the larger square stimulus and at small disparity values (Experiment 2). (3) It could be shown in a supplemental analysis of the results of Experiment 2 that the stimulus processing which underlies depth perception can be described by means of a cancellation model. This assumed process of neutralizing is due to the combination of the afferent disparity message with the internal distance message in such a way that the dependence of disparity on viewing distance can be redissolved.

\section{EXPERIMENT 3}

In order to establish the validity of the difference measure used for perceived depth in Experiments 1 and 2, the relationship between the subject's distance perception and the magnitude of the physical distance in the range investigated in these experiments was analyzed. Therefore, it was first determined how well subjects were able to compare the perceived position of the fixation target, which was presented between 60 and $180 \mathrm{~cm}$, with the subsequent probe. Then, by applying a ratio-production method, the relationship between perceived and physical distance under the experimental conditions used here was examined.

\section{Method \\ Subjects. The same six students as in Experiment 1 participated as subjects.}

Apparatus and Stimuli. The same experimental arrangement as in Experiments 1 and 2, but without the presentation of the stereoscopic stimuli was also similar: The fixation stimulus (duration: $2.2 \mathrm{sec}$ ) was followed by the comparison stimulus (duration: $2 \mathrm{sec}$ ) after an interval of $1 \mathrm{sec}$. The distance of the fixation target served as the independent variable, being varied according to the following gradation: $60,90,120,150$, and $180 \mathrm{~cm}$.

Procedure. The subjects had to solve four different tasks. The first task was to let the experimenter move the comparison stimulus between successive trials until the perceived distance of the comparison stimulus corresponded with that of the fixation target (equidistance judgment). The subject began a trial by looking at the fixation target and remembering its distance position. Then the subject also glanced at the comparison stimulus and judged its distance; according to the result of this comparison, he instructed the experimenter to move the probe. After approximately four to eight settings, a final trial was reached in which the subject saw the fixation target and the comparison stimulus at exactly the same distance. The position of the probe was then read off and used as the measurement value.

Using a similar procedure, the other tasks required that the subjects set the comparison stimulus at $1 / 3,1 / 2$, and $2 / 3$ of the judged distance of the fixation target $(1 / 3,1 / 2$, and $2 / 3$ judgment). By comparing the results of these three procedures, it was determined if the subject always used the same perceptual distance scale.

For each of the five distance values, four measurements were made in each task and for each subject. The order of presentation of the distance and task conditions were varied across the subjects.

\section{Results}

(1) The means of the equidistance judgments comprised a linear function of the fixation distance with a slope of 1 ; the standard deviations were small, lying between .6 and $.9 \mathrm{~cm}(\mathrm{~N}=6)$. Therefore, in the range of distance values examined, the subjects were able to map the perceived distance of the comparison stimulus with the distance of the previously presented fixation target quite exactly.

(2) Figure 8 shows the means and standard deviations of the distance settings as a function of the fixation distance and the three different tasks, the $1 / 3,1 / 2$, and $2 / 3$ judgments. The means proved to

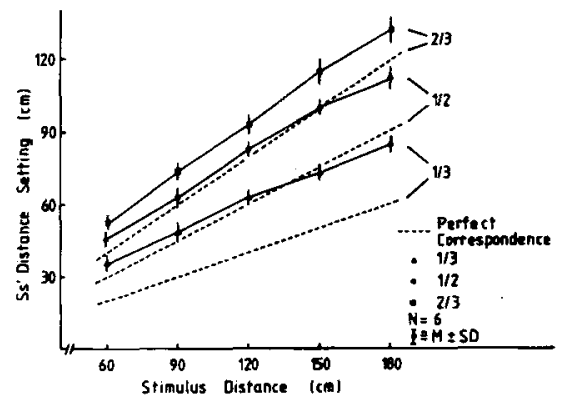

Figure 8. Subjects' distance settings as a function of stimulus distance for the $1 / 3,1 / 2$, and $2 / 3$ judgments; means and standard deviations for six subjects. The dashed line corresponds to a perfect task solving. 
be linear functions of the stimulus distance, but their values systematically differ from those which could be expected if there were a perfect correspondence between physical and perceived distance. When, for example, the distance interval of $180 \mathrm{~cm}$ was bisected, then the distance between the subject's eyes and the position of the $1 / 2$ judgment measured $109 \mathrm{~cm}$ instead of $90 \mathrm{~cm}$ : the more distant interval was subjectively judged to be smaller than it really was.

(3) Supposing that subjects divided the presented distance intervals according to the prescribed ratios, then the relation between physical and perceived distance could be derived from these results (cf. Torgerson, 1958). Figure 9 shows this relationship for the distance range investigated. These values could be approximated by a linear function: $\mathrm{D}^{\prime}=$ $-6.76+.87 \mathrm{D}\left(\mathrm{r}^{2}=.99 ; \mathrm{N}=15\right)$. The subjects therefore apparently used the same distance scale for all three ratio-production tasks.

These results agree, for example, with those of Mayer-Hillebrand (1932), who showed that between 40- and $200-\mathrm{cm}$ distances intervals were underscored, but were linearly related to physical distance. (Above and below this range the relationship appeared to be curvilinear. Therefore the $y$ intercept in Figure 9 is not interpreted.) The linear relationship found justified using a measure of perceived depth derived from the difference in the distance positions of the probe set for the fixation target and the stereoscopic stimuli. According to the slope of the above relation, this difference had to be weighted with a factor of 87 .

It is assumed that subjects can match the perceived position of the comparison stimulus with that of the stereoscopic stimuli in such a way that the position of these can be measured and the difference between the settings for the fixation target and the stereoscopic stimuli can be interpreted according to the above distance scale. Applying this procedure of measurement, eye convergence presumably functioned as the main source of egocentric distance (cf. Gogel, 1977).

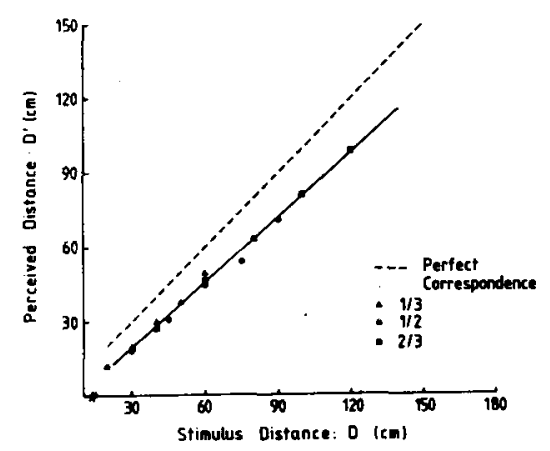

Figure 9. Perceived distance as a function of stimulus distance as derived from the data of Figure 8 . The dashed line indicates a perfect correspondence between both variables.

\section{REFERENCE NOTES}

1. von der Heydt, R., \& Adorjani, C. Orientation disparity versus position disparity: Different stereoscopic mechanisms. Paper presented at the 19th meeting of the German Experimental Psychologists, Konstanz, 1977.

2. Kalveram, K. Th. Das Marburger System. 1. Teil: Das Digital-System. Berichte aus dem Institut für Psychologie der Philipps-Universität Marburg, No. 44, 1975.

\section{REFERENCES}

Bischоғ, N. Psychophysik der Raumwahrnehmung. In W. Metzger (Ed.), Handbuch der Psychologie, Allgemeine Psychologie, Wahrnehmung und Bewusstsein (Bd. I/1). Gottingen: Hogrefe Verlag, 1966.

Bischof, N. Optic-vertibular orientation to the vertical. In H. H. Kornhuber (Ed.), Handbook of sensory physiology; Vestibular system, Part 2: Psychophysics, applied aspects and general interpretations (Vol. VI/2). Berlin, Heidelberg, New York: SpringerVerlag, 1974.

BIsHOP, P. O. Neurophysiology of binocular vision and stereopsis. In R. Jung (Ed.), Handbook of sensory physiology; Central processing of visual information (Vol. VII/3 A). Berlin, Heidelberg, New York: Springer-Verlag, 1973.

BLAKEMORE, C. The range and scope of binocular depth discrimination in man. Journal of Physiology (London), 1970, 211, 599-622.

Blakemore, C., Fiorentini, A., \& Maffei, L. A second neural mechanism of binocular depth discrimination. Journal of Physiology (London), 1972, 226, 725-749.

FolEy, J. M., \& Richards, W. Effects of voluntary eye movement and convergence on the binocular appreciation of depth. Perception \& Psychophysics, 1972, 11, 423-427.

GOGEL, W. C. The metric of visual space. In W. Epstein (Ed.), Stability and constancy in visual perception: Mechanisms and processes. New York: Wiley, 1977.

Gulick, W. L., \& LAWson, R. B. Human stereopsis: A psychophysical analysis. New York: Oxford University Press, 1976.

Mayer-Hillebrand, F. Über die scheinbare Entfernung oder Sehtiefe. Zeitschrift für Sinnesphysiologie, 1932, 63, 38-86.

ONo, D. A., \& Cumerford, J. Stereoscopic depth constancy. In W. Epstein (Ed.), Stability and constancy in visual perception: Mechanisms and processes. New York: Wiley, 1977.

OWENS, D. A., \& LeIBowitz, H. W. Oculomotor adjustments in darkness and the specific distance tendency. Perception \& Psychophysics, 1976, 20, 2-9.

Pettigrew, J. D., Nikaka, T., \& Bishop, P. O. Responses to moving slits by single units in cat striate cortex. Experimental Brain Research, 1968, 6, 373-390.

RichaRDs, W. Stereopsis and stereoblindness. Experimental Brain Research, 1970, 10, 380-388.

RichaRds, W., \& KAYE, M. G. Local versus global stereopsis: Two mechanisms? Vision Research, 1974, 14, 1345-1347.

RITTER, M. Effect of disparity and viewing distance on perceived depth. Perception \& Psychophysics, 1977, 22, 400-407.

TORGERson, W. S. Theory and methods of scaling. New York: Wiley, 1958.

HoFsten, C. von. The role of convergence in visual space perception. Vision Research, 1976, 16, 193-198.

Wallach, H., \& Zuckerman, C. The constancy of stereoscopic depth. American Journal of Psychology, 1963, 76, 404-412.

Westheimer, G., \& Tanzman, I. J. Qualitative depth localization with diplopic images. Joumal of the Optical Society of America, 1956, 55, 116-117.

(Received for publication April 3, 1978; revision accepted December 1,1978 .) 